\title{
XII.
}

Aus dem allgemeinen Krankenhaus Hamburg-Eppendorf.

\section{Primäre Seitenstrangdegeneration und Meningitis cervico- dorsalis levis als anatomische Grundlage in 2 Fällen von „Syphilitischer Spinalparalyse"}

Von

\section{Nonne. \\ (Mit Tafel IV.)}

Ich habe mich bereits mehrere Male mit der von Erb als klinisches Krankheitsbild aufgestellten "syphilitischen Spinalparalyse" beschäftigt, und zwar im Jahre 1896, 1902 and 1905.

1896 handelte es sich einmal um den Fall einer Kombination diffuser myelitischer Prozesse mit einer primären Seitenstrangdegeneration, ein ander Mal $\mathrm{nm}$ einen Fall primärer kombinierter Strangdegeneration in den Py-Str. und KlHS-Str. einerseits, den Gollschen Strängen andererseits; 1902 referierte ich die bis dahin vorliegende Literatur, und 1905 kounte ich dem erst 1903 wieder von Erb revidierten Material einen weiteren Fall beifügen. In diesem Fall, den ich durch 14 Jahre beobachtet hatte, war das klinische Symptombild der Muskelspannungen mit erhöhten Sehnenreflexen und geringer motorischer Schwäche ohne sonstige Anomalien am Nervensystem anatomisch bedingt durch eine geringe fleckweise "myelitische" Degeneration im Dorsalmark, kombiniert mit einer primären Degeneration in den Gollschen Strängen des Halsmarks und oberen Dorsalmarks sowie einer Degeneration des PyS-Str. im Lendenmark, neben einer nicht spezifischen Wandverdickung der Gefässe im Rückenmarksquerschnitt, einer Endarteriitis proliferans der Arteria spinalis anterior und einer leichten Meningitis posterior cervicalis et dorsalis. Seither ist in der Literatur, soweit ich ersehen kann, über dies Thema nicht wieder verhandelt und speziell auch kein weiterer anatomischer Beitrag geliefert worden. ${ }^{1}$ ) Es ist leicht begreiflich, dass die Gelegenheit, anatomische Untersuchungen an lange Zeit hindurch beobachteten Fällen vorzunehmen, sich nur selten bietet: dazu gehört entweder, wie es in

1) Anmerkung bei der Korrektur: Inzwischen ist in dieser Zeitschrift die schöne Arbeit von Wimmer aus Kopenhagen erschienen, auf deren Inhalt ich bier nur zu verweisen kann.

Deatsche Zeitschrift f. Nervenheilkunde. 33. Bd. 
2 meiner bisherigen 3 Fälle war, dass ein Privatpatient lange in Beobachtung bleibt und dass dann die Sektion ermöglicht ist, oder es muss der Kranke dauernd nnter der Kontrolle einer und derselben Krankenhausabteilung bis zum Schluss bleiben.

Dies letztere war bei den heute zu besprechenden Kranken der Fall gewesen: 8 Jahre hindurch habe ich den einen Kranken mit kürzeren und längeren Unterbrechungen in Eppendorf unter Augen gehabt, und ein glücklicher Zufall fügte es, dass ich, als er das letzte Mal moribund mit einer Apoplexia cerebri auf eine andere Abteilung ins Krankenhaus kam, ich in Hamburg anwesend war und die Leiche erkannte, deren Hirn und Rückenmark mir - wofür ich meinem Kollegen Herm. Dr. Sick auch hier bestens danke - dann überlassen wurde. 'Es ist dies einer jener 8 Fälle, von denen ich 1905 sagte, dass ich sie "seit langen. Jabren beobachte". Von den von mir als „reine", in Erbschen Sinn beobachteten Fällen sind nun 3 zur anatomischen Untersuchung gekommen.

Und im zweiten Fall fügte es sich ebenfalls glücklich, dass ich, nachdem ich Patientin 9 Jahre lang nicht gesehen hatte, im Krankenhaus in die Lage kam, die Kranke noch einmal eingehend beobachten und untersuchen und dann die anatomische Untersuchung machen zu können.

Fall 1. Der Eisenbahnbeamte St. war 5 mal im Eppendorfer Krankenhause aufgenommen. Ich hatte denselben dort unter Beobachtung vom 15. XI. 1898 bis Ende 1903. Vorher war er während des August 18939 Tage im Krankenhaus in Behandlung gewesen. Er stammt aus gesunder Familie, 1 Bruder war Potator strenuus; er selbst war als Kind im wesentlichen gesund, nur hatte er mit 12 Jahren Pocken gehabt. Er hatte 6 Jahre vor seiner ersten Aufnahme im Krankenhaus, d. h. im 25. Lebensjahr, einen syphilitischen Schanker acquiriert and hatte hinterher Sekundärerscheinungen gehabt. Bei seiner ersten Aufnahme ins Krankenhaus klagte er inber leichte ziehende Schmerzen in den unteren und oberen Extremitäten, auch gab er an, im letzten Jahre $a b$ und $z u$ epileptische Anfälle gehabt zu haben. Es wurde damals eine objektive Anomalie weder an den inneren Organen noch am Nervensystem festgestellt. Patient wurde nach 9 Tagen Krankenhausaufenthalt beschwerdefrei entlassen.

Bei seiner 2. Aufnahme (1898) klagte er über Gefühl von Schwäche im rechten Arm und rechten Bein, in geringerem MaBe auch in den linksseitigen Extremitäten.

Die Beschwerden waren aufgetreten, nachdem er 3 Wochen lang täglich Bäder in der Ostsee genommen hatte. Die Untersuchung ergab damals, dass das Herz und Lungen normal waren, auch die Abdominalorgane sich normal verhielten. Seitens des Nervenensystems fand sich ein paretischer Zustand in den unteren Extremitäten, und zwar waren am Bein die Benger am Oberschenkel und die Strecker am Unterschenkel schwächer als die der entsprechenden Antagonisten. Beim Gehen rechts leichtes Nach- 
schleifen. Aher auch in den oberen Extremitäten war die Kraft nicht normal. Die Sensibilität war am ganzen Körper normal. Die Sehnenreflexe waren beiderseits lebhaft, die Hautreflexe beiderseits normal. Bei passiven Bewegungen bestand geringe Spannung in den unteren Extremitäten, rechts mehr als links. Seitens der Gehirnnerven bestand keine Anomalie; keine oculopupillären Anomalien, der Augenhintergrund beiderseits normal, sensorische Funktionen intakt, keine Intelligenzstörung. Bei seiner Entlassung, die nach 4 Wochen erfolgte, befand er sich subjektiv gebessert. Der objektive Status war derselbe, nur dass jetzt rechterseits Patellarklonus angedeutet und Achillesklon us in typischer Weise vorhanden war.

Im Jahre 1900 kam Patient abermals zur Aufnahme. Er hatte öfter inzwischen an Schwindelzuständen gelitten. Er klagte über Abnahme der Potenz; die Gehfähigkeit war etwas zurückgegangen.

Es zeigte sich auch jetzt, dass eine leichte spastische motorisehe Parese an beiden unteren Extremitäten bestand, dass beiderseits ausgesprochener Patellar- und Fussklonus nachweisbar war, rechts noch stärker als links, dass die Sehnen- und Periostreflexe an den in geringem Grade rigiden oberen Extremitäten bei etwas herabgesetzter motorischer Kraft sehr lebhaft waren, dass die Hautreflexe sich normal verhielten. Die Sensibilität war in allen Qualitäten völlig intakt. Der Gang war leicht spastisch, rechts wurde das Bein stärker nachgeschleift als links; keine statische und lokomotorische Ataxie; kein Romberg; kein Tremor. Die Gehirnnerven waren sämtlich intakt, keine oculopupillären Anomalien; ophthalmoskopischer Befund normal, sensorische Funktionen intakt, Psyche und Intelligenz normal, keine Sprachstörung.

Patient verliess schon nach 1 Woche wieder das Krankenhaus und kam 3 Jahre später (1903) ron neuem zur Aufnahme. Er hatte inzwischen seinen Dienst als Stationsgehilfe bei der Bahn versehen, klagte aber jetzt über immer mehr zunehmende Schwäche in den Beinen und Armen. Am Tage vor seiner Aufnahme hätte er einen Anfall von Kopfschmerzen, Übelkeit und Schwindel bekommen, auch nähme sein Gedächtnis ab. Der objektive Status batte sich seit der letzten Untersuchung nicht verändert, nur hatte die spastische Parese des rechten und in geringerem Grade auch des linken Beines etwas zugenommen. Während seines jetzt zirka 6 Monate dauernden Aufenthalts klagte er oft über Kopfschmerzen und Schwindel and öfter über Kreuzschmerzen. Im übrigen blieb der Zustand unverändert, speziell wurden irgend welche Anomalien am Augenhintergrund und an den Augenmuskeln nicht festgestellt. Die Diagnose, die bisher geschwankt hatte zwischen syphilitischer Spinalparalyse und multipler Sklerose, wurde jetzt, nachdem sich in den 5 Jahren nichts für multiple Sklerose Charakteristisches hinzugesellt hatte, fixiert auf "syphilitische Spinalparalyse".

Der Zustand verschlechterte sich dann langsam, wie ich nachträglich von seinen Verwandten hörte, und seit 2 Jahren war er nicht mehr imstande gewesen, seiner Arbeit nachzugehen und hatte auch öfter über Kopfschmerzen und Schwindel geklagt.

Zum letzten Male kam Patient $1 \mathrm{Jahr}$ später zur Aufnahme und zwar diesmal in schwer benommenem Zustand. Es handelte sich um die Zeichen einer schweren Apoplexia cerebri sanguinea. Patient war völlig benommen. Der Kopf stand in leichter $Z$ wangsstellung nach rechts, die Bubi waren 
unbeweglich, die rechte Pupille war weiter als die linke, beide leicht entrundet und bei konzentrierter Belichtung starr; ophthalmoskopisch beiderseits normaler Befund. Kontrakturen in allen 4 Extremitäten; die Sehnenreflexe waren überall gesteigert, beiderseits Babinski, Bauchdecken- und Kremasterreflexe beiderseits aufgehoben. Die Atmung war röchelnd und verlangsamt.

Patient kam nicht wieder zu sich, sondern starb bereits 8 Stunden nach seiner Aufnahme.

Es handelt sich hier um einen Mann, der mit 25 Jahren sich syphilitisch infiziert hatte, bei dem sich zuerst 6 Jahre nach der Infektion Zeichen von spastischer Parese in den unteren Extremitäten, zunächst mit Bevorzugung der rechten Seite geltend machten, bei dem dieser spastisch-paretische Zustand auch in den oberen Extremitäten, wenngleich in geringerem Grade, ausgesprochen war, und der in den letzten Jahren seines Lebens wegen dieser motorischen Störung in den Extremitäten zur Arbeitslosigkeit verurteilt war. Andere Störungen seitens des Nervensystems bestanden nur in den letzten 2 Jahren, und zwar in Gestalt von Kopfschmerz und Schwindel. Objektiv fand sich nichts weiter als der spastische Symptomenkomplex an den 4 Extremitäten. Der Tod erfolgte', unter den Erscheinungen einer Hirnapoplexie.

Bei der Sektion ergab sich als Wesentliches: mässige Myodegeneratio fibrosa cordis, mässige Arteriosklerose der Koronararterien, starke Arteriosklerose der Art. basilaris und der Arterien des Circulus arteriosus Willisii, chronische Leptomeningitis basalis und doppelseitige Orchitis fibrosa, glatte Atrophie des Zungengrundes.

Die Art. basilaris war in ihrer Mitte aneurysmatisch erweitert und an einer kleinen Stelle perforiert. Die Hirnbasis war mit frischen und älteren Blutmassen bedeckt. Die Seitenventrikel waren etwas erweitert und enthielten wenig frisches Blut. Die Pia mater war an der Konvexität und an der Basis leicht getrübt und verdickt. Das Hirn, auf zahlreichen sagittalen und frontalen Durchschnitten nach 6 wöchentlicher Härtung in Formol, war makroskopisch ohne jede Veränderungen.

Das Rückenmark zeigte an der Hinterfläche in seiner ganzen Ausdehnung und am meisten im Dorsalteil leichte Verdickung und Trübung der Pia. Auf Querschnitten erkennt man schon am frischen Präparat eine deutliche graue Färbung der Gegend der Pyramidenseitenstränge beiderseits. Im übrigen liess das R.-M. makroskopisch eine Anomalie nicht erkennen.

Die mikroskopische (Härtung in Sol. Müller, in Formol, Färbung nach Weigert, Weigert-Pal, van Guison) Untersuchung ergab:

Halsmark ( 4 Höhen): In der hinteren Zirkumferenz ist die Pia mater etwas verdickt und gewuchert; die hinteren und vorderen Wurzeln sind intakt, die Gefässe in den hinteren Wurzeln zeigen erhebliche Verdickung der Wände, am meisten ist die Intima verdickt. Die vordere und hintere graue Substanz erscheint intakt an Zellen und markhaltigen Fasern. In der weissen Substanz sind die PyS-Str. beiderseits stark sklerosiert, 
nach vorn $\mathrm{zu}$ erstreckt sich eine Randdegeneration, die, nach vorn $\mathrm{zu}$ sich verschmächtigend, bis an die vordere Medianfissur reicht; im übrigen sind die V.-Str. intakt. Die H.-Str. lassen keine Anomalien erkennen.

Die Art. spinalis ant. und posterior zeigt normale Verhältnisse. Dorsalmark (4 Höhen): Die meningitische Verdickung der Pia ist etwas stärker als im Halsmark, die vorderen und hinteren Wurzeln sind auch hier intakt; eine pathologische Wandverdickung der Gefässe der hinteren Wurzeln ist nicht zu erkennen, die vordere and hintere graue Substanz ist normal. Die Sklerose in den S.-Str. nimmt die ganzen PyS-Str. ein, greift nur ganz wenig ins Gebiet der S.-Str.-Reste über, nach vorn ins Gebiet der KIHS-Str., und verschmächtigt sich bis dicht an die vordere Medianfissur in Form einer Randdegeneration. Die Vorderstränge sind im übrigen normal, die H.-Str. sind normal. Die Art. spinalis ant. und post. ist ebenfalls normal.

Lendenmark (3 Höhen):

Eine pathologische Verdickung der Pia mater ist nicht vorhanden. Die vorderen und hinteren Wurzeln sind intakt, die Wandverdickung der Gefässe in den hinteren Wurzeln ist sehr ausgesprochen. Die vordere und hintere graue Substanz zeigt keine Anomalien, die Sklerose in den S.-Str. beschränkt sich ausschliesslich auf das Areal der PyS-Str., von einer Randdegeneration ist nichts zu sehen. Die Vorderstränge sind normal, ebenso die Hinterstränge. Die Art. spinalis ant. et post. ist intakt.

In der Medulla oblongata sind die Pyramiden erheblich gelichtet, die sensiblen Teile der Medulla oblongata, die Kerngebiete und die von den Kernen das verlängerte Mark durchziehenden motorischen Nervenfasern sind intakt; ebenso sind die zum Kleinhirn gebörenden Bahnen nicht verändert.

Vom Gehirn wurde mikroskopisch untersucht je 1 Stück der linken 3. Stirnwindung und der linken vorderen Zentralwindung (WeigertNissl). Es fanden sich an der Anordnung der verschiedenen Zellgruppen, an den Zellen selbst keine nennenswerten Veränderungen; ebenso erschienen die markhaltigen Fasern in der Projektionsstrahlung, in der Zonula, den Tangentialstreifen, dem Bechterewschen Streifen, dem supraradiären Netzwerk, dem Baillargerschen Streifen sowie in der 1. und 2. Meynertschen Windung normal.

Wir haben es hier somit zu tun mit einer chronischen Sklerose in den S.-Str. des R.-M.s; die Erkrankung der S.-Str. betrifft die PySStr. und dehnt sich anf die KlHS-Str. aus; ausserdem besteht eine Randsklerose, rechts stärker ausgeprägt als links; die Vorderstränge sind, abgesehen von einer geringen Randsklerose, ebenso wie die Hinterstränge frei von Erkrankung. Die graue Substanz ist in den Vorder- und Hintersträngen intakt, im Hals- und Dorsalmark findet sich eine chronische Meningitis posterior geringen Grades; die Gefässe im R.-M.-Querschnitt zeigen nichts weiter als eine mässige Verdickung der Wandungen, die Art. spinalis anterior et posterior ist normal, die Gefässe in den hinteren Wurzeln zeigen erhebliche Wandverdickung. 
Die Erkrankung der Py-Bahnen erstreckt sich bis in die Medulla oblongata hinein, während die übrigen Teile der Medulla oblongata und speziell auch die Kerngebiete intakt sind.

Der sonstige Sektionsbefund interessiert dadurch, dass wir Residuen von Lues finden, die wir als spezifisch auffassen müssen: das ist erstens die glatte Atrophie des Zungengrundes und zweitens die doppelseitige Orchitis fibrosa bei einem Manne, der andere Ursachen für diese Hodenschwielen nicht aufzuweisen hatte. Es kommt hinzu die für das Alter von nur 39 Jahren hochgradige Arteriosklerose der Hirnarterien.

Es handelt sich in diesem Falle um eine primäre Erkrankung der Py-Bahnen in den Seitensträngen; ich glaube, dass das geringe Hineinreichen der Erkrankung in das Gebiet der KlHS-Str. nicht dazu berechtigt, diesen Fall zu der Kategorie der „kombinierten Strangerkrankungen" zu rechnen; denn, wie die Photographien zeigen, ist in einzelnen Höhen des R.-M.s die Degeneration der PyS-Str. eine streng "systematische", und in den Höhen, in denen das Areal der KIHS-St. mitbeteiligt ist, ist keineswegs das ganze Gebiet derselben ergriffen, und die vorhandene Randdegeneration gestattet die geringe Erkrankung im Gebiet der KlHS-Str. anf ihr Konto zu setzen.

Ich rechne den Fall somit zu der 3. Kategorie unter den 4 verschiedenen Kategorien, die wir bis heute als anatomische Grundlage der klinischen Bilder der "syphilitischen Spinalparalyse" kennen; das sind:

1. chronische fleckweise Myelitis transversa mit auf- und absteigender Degeneration;

2. eine solche kombiniert mit einer primären Py-Str.-Degeneration;

3. Py-Str.-Degeneration allein;

4. eine kombinierte Strangerkrankung, d. h. eine in den H.-Str. und in den S.-Str. (PyS-Str., KlHS-Str., eventl. auch Gowers sche Str.) primär auftretende Erkrankung.

Wir können somit von diesem neuen Fall sagen:

Die klinische Beobachtung erstreckte sich über eine längere Reibe von Jahren unter den Augen desselben Beobachters. Andere Symptome als solche, die wir auf die S.-Str. im klinischen Sinne zu beziehen gewohnt sind, wurden niemals beobachtet. Die Anamnese auf Lues war positiv. Die Sektion ergab syphilitische und parasyphilitische Befunde an den inneren Organen und eine fast ausschliessliche Erkrankung der PyS-Str. im Rückenmark.

Ich glaube hiernach, dass auch dieser Fall als einwandsfrei gelten kann. Den stark ausgeprägten Symptomen entsprach auch eine starke Degeneration in den entsprechenden Rückenmarksbahnen, während dem viel weniger stark betonten Symptombild in meinem Falle 1 aus dem 
Jahre 1896 und dem noch weniger stark ausgesprochenen Fall aus dem Jahre 1905 nur eine geringe resp. sehr geringe Erkrankung in den Seitensträngen entsprach.

Dass hier keine Paralyse und nicht die Residuen einer chronischen Meningomyelitis syphilitica sowie auch keine spastische Form der amyotrophisehen Lateralsklerose vorlagen, ergibt sich aus der Krankengeschichte und ans dem mikroskopischen Befund an Hirn und Rückenmark ron selbst.

Fall 2. Das 25 jährige Fräulein R. sah ich am 25. November 1897 zuerst mit ihrem Hausarzt, Herrn Dr. Crone, zusammen. Sie war neuropathisch belastet insofern, als die Mutter und die Mutter der Mutter psychotisch gestorben waren und als der Vater ein pathologisch jähzorniger Mann war.

Als Kind und als junges Mädchen war sie im wesentlichen stets gesund gewesen. Sie konsultierte mich wegen einer lältmungsartigen Schwäche der. oberen und unteren Extremitäten, die seit 11/2 Jahren sich langsam entwickelt hatte. Diese Läbmung hatte vor $1 \mathrm{Jahr}$ ihren Höhepunkt erreicht, so dass sie damals nicht gehen und nicht stehen konnte und auch gefüttert werden musste; auch hatten damals in tensive Nackenschmerzen bestanden.

Die nähere Nachfrage ergab, dass es sich um eine extragenitale Syphilis infeltion handelte: Patientin war vor 3 Jahren verlobt gewesen und hatte von ihrem Bräutigam einen Lippenschanker acquiriert; die Verlobung wurde dann später aufgehoben wegen "schlechten Lebenswandels" des Bräutigams. Patientin liess sich aber nicht sachgemäss behandeln, sondern gebrauchte Naturheilkunde und allerlei sonstige nicht spezifische, von seiten Fremder und Bekannter angeratene Behandlungsarten. Zirlia 3 Monate nach der Infektion trat eine Hornhautentzündung und Iritis auf dem rechten Auge auf, die unter Hg-Injektionsbehandlung (Dr. du Mesnil-Altona) heilte; ein halbes Jahr später entwickelte sich ein tiefes Ulcus am r. Unterschenkel, das einer sachgemässen Behandlung: wieder unterzogen wurde und ausheilte. Als die Lähmungen zuerst sich bemerkbar machten, ging Patientin wieder zum „Naturheilkundigen", der das Leiden für eine "Folge der Hg-Kur" erklärte.

Ich fand: spastische Parese beider oberen Extremitäten, d. h. motorische Schwäche in allen Gebieten der oberen Extremitäten mit Spannungen bei passiven und aktiven Bewegungen, keine Sensibilitätsstörungen; in den unteren Extremitäten ebenfalls spastische Parese mit pathologischer Erhöhung der Sehnenreflexe (Patellarklonus, Achillesklonus), keine Sensibilitätsstörungen; geringe Blasenschwäche; die elektrische Erregbarkeit war normal; Fehlen aller cerebralen und bulbären Symptome; die inneren Organe nicht nachweislich affiziert.

Unter einer Kombination von Quecksilber-Jodbehandlung besserten sich die Paresen und die Spannungen. Ich sah Patientin zunächst zum letzten Mal 1/2 Jahr nach meiner ersten Untersuchung und konstatierte eine quantitative Besserung der Symptome an den oberen und unteren Extremitäten; die Blasenbeschwerden waren zurückgegangen.

Ich sah Patientin dann 9 Jahre nicht wieder. Sie kam dann ins Krankenhaus in wesentlich verschlechtertem Zustand. Ich erfuhr, dass die 
frühere Besserung nur 1 Jahr angehalten hatte und dass die Verschlechterung allmählich dann eingesetzt hatte. Sie hatte sich keiner spezifischen Kur inzwischen unterzogen.

Die Untersuchung ergab jetzt auf meiner Abteilung, dass die linke Pupille etwas weiter war als die rechte, links oval verzogen, leicht mydriatisch, auf Licht und Konvergenz nicht reagierend; rechts etwas myotisch, auf Licht schwach, auf Konvergenz normal reagierend, der Augenhintergrund normal, keine Anomalien im Bereich der Funktion der Bulbomotoren, speziell kein Nystagmus. Die äbrigen Hirnnerven waren sämtlich intakt, die Sprache war normal, die Nackenmuskulatur intakt. Im Bereich der oberen Extremitäten fand sich eine erhebliche Parese in allen Muskeln der Ober- und Unterarme und Hånde. Ausserdem bestand eine allgemeine Muskelmacies, die Sehnen- und Periostreflexe waren pathologisch gesteigert. Die Untersuchung der Sensibilität ergab nichts weiter als eine leichte Störung des Lagegefühls in den Fingern, während die anderen Qualitäten der Sensibilität intakt waren. Es bestand auch Parese und Vermehrung des Muskeltonus in den Bauchmuskeln. Sensibilitätsstörungen waren am Rumpf nicht deutlich nachzuweisen. An den unteren Extremitäten bestand ebenfalls neben einer allgemeinen Muskelabmagerung starke Spastik, und zwar waren die Beine in den Knieen gebeugt und setzten passiven Bewegungsversuchen starken Widerstand 'entgegen. Es bestand Patellar- und Achillesklonus beiderseits, deutliches BabinskiOppenheim- und Strümpellsches Phänomen; Plantarreflex beiderseits erhalten. Auch in den Zehen und Füssen war das Lagegefühl gestört, während die übrigen Qualitäten der Sensibilität keine sicheren Störungen erkennen liessen. Die elektrische Erregbarkeit ergab an den aberen und unteren Extremitäten eine der Muskelabmagerung entsprechende Herabsetzung für den faradischen Strom, ohne sonstige Veränderungen der quantitativen Reaktion für den faradischen und quantitativen und qualitativen Reaktion bei galvanischem Strom.

Patientin blieb in diesem Zustand unverändert. Eine nochmalige energische Behandlung mit Schmierkur und Jod hatte keinen Einfluss. Sie kam schon mit Decubitus am Kreuzbein anf. Der Decubitus vergrösserte sich allmählich, griff in die Tiefe, es bestand ausserdem starke Cystopyelitis, und nach zirka 4 monatlichem Krankenhausaufenthalt ging Patientin an Sepsis zugrunde.

Die Diagnose hatte eine Zeitlang geschwankt zwischen Paralysis spinalis syphilitica und Sclerosis multiplex, hatte sich zuletzt jedoch der ersteren Annahme mehr zugeneigt wegen der Pupillenanomalien und wegen des dauernden Fehlens von sonstigen oculären Anomalien.

Die Sektion ergab keine wesentlichen Anomalien an den Brust- und Baucheingeweiden, nur die Zeichen einer frischen Sepsis als Folge einer Cystopyelitis und eines schweren gangränösen Decubitus.

Die Sektion des Gehirns ergab: Dura mater normal, das Gehirn von aussen betrachtet normal, keine nennenswerte Arteriosklerose an den Gefässen, in der Höhe der Konvexität deutliche Trübung und Verdickung der Pia, die jedoeh ohve Substanzverlust abziehbar ist; die Ventrikel sind erweitert, das Ependym zart. Zahlreiche Frontal- und Sagittalschnitte durch das Grosshirn lassen keine Herde irgend welcher Art erkennen. 
Primäre Seitenstrangdegeneration u. Meningitis cervico-dorsalis levis etc. 169

Die Rückenmarkssektion ergab, dass die Dura mater normal ist, die Leptomeninx hingegen auf der ganzen Hinterfäche getrübt und verdickt erscheint; das Rückenmark erscheint im ganzen schmächtig und zeigt schon auf frischen Querschnitten graue Verfärbung in äer ganzen Ausdehnung der Seitenstränge.

Die PyS-Str. sind streng symmetrisch vom Halsmark bis hinunter ins Lendenmark degeneriert. Die Degeneration nimmt genau das Areal der PyS-Str. ein and im oberen und mittleren Halsmark schliesst sich nach vorn von der genannten Degeneration noch ein schmaler Saum an, der sich marginal bis an die Grenze der Vorderstränge hinzieht.

Die mikroskopische Untersuchung zeigt, dass es sich um eine chronische Sklerose handelt, in der die Gefässe nicht erkrankt sind, sondern nur die übliche, nicht spezifische. Wandverdickung aufweisen.

Es zeigt sich ferner, dass die Pia mater am Hals- und Dorsalteil an der Hinterseite der Peripherie etwas verdickt ist (Leptomeningitis chronica).

In den Hintersträngen und in den Vordersträngen lässt sich keine Anomalie feststellen; die vordere und hintere graue Substanz ist normal, die hinteren und vorderen Wurzeln sind intakt.

Vom oberen Halsmark weiter nach oben hin verliert sich die Erkrankung bald, so dass oberhalb der Pyramidenkreuzung nichts mehr von Sklerose zu sehen ist.

Zusammengefasst handelt es sich in diesem zweiten Falle um eine extragenitale (Lippen) syphilitische Infektion bei einem Mädchen, die bereits $1 \frac{1}{2}$ Jahre nach der Infektion spinale Symptome zeigte. Es entwickelte sich zunächst der reine "spastische Symptomenkomplex", der unter einem Traitement mixte vorübergehend eine quantitative Reduktion erfuhr. 9 Jahre später hatte sich der spastische Symptomenkomplex sehr hochgradig verstärkt, hinzugetreten waren doppelseitige Pupillenanomalien.

Die anatomische Untersuchung des Zentralnervensystems ergab eine auf die PyS-Str. beschränkte systematische und symmetrische Degeneration chronischen Charakters mit leichter Leptomeningitis spinalis posterior bei intaktem Hirn.

Auch dieser Fall gehört somit zur Kategorie 3 der 4 oben aufgezählten Kategorien.

Ich meine, dass diese 2 Fälle auch für die Wertigkeit der spinalen Pyramidenbahnen beim Menschen ein gewichtiges Wort reden und dass sie geeignet sind, vor einer Verallgemeinerung der interessanten experimentellen Ergebnisse Rothmanns zu warnen.

Ich habe oben auseinandergesetzt, warum nur selten einwandsfreie Fälle der Literatur einverleibt werden können; dass dies aber bei grösserem Beobachtungsmaterial demjenigen, der sich für diese Frage interessiert, doch möglich ist, beweisen meine Fälle, die jetzt auf 5 angewachsen sind. Hoffentlich finden meine Beobachtungen 
auch von anderer Seite Bestätigung. Auf die Literatur einzugehen, habe ich keine Veranlassung, da, soweit ich sehen kann, seit meiner Veröffentlichung im Jahre 1905 keine einschlägigen Beobachtungen publiziert sind, und ich das Wesentliche aus der Literatur in meinen früheren Arbeiten angeführt habe.

\section{Erklärung der Abbildungen auf Tafel IV.}

Fall 1.

Fig. a. C.-W. 3. Verdickung der Pia mater in der ganzen Zirkumferenz; im r. PyS-Str. und etwas weiter nach vorn greifend Degeneration; links ist die Degeneration im S.-Str. mehr diffus.

Fig. b. D.-W. 1. Der rechte PyS-Str. ist ganz degeneriert, nach vorn zu eine marginale schwache Degeneration, links dieselbe Lokalisation der Degeneration, aber quantitativ geringer ausgesprochen.

Fig. c. D.-W. 5. Verdickung der Leptomeninx an der hinteren Peripherie.

Fig. d. D.W.11. Dasselbe Bild der Degeneration; Leptomeningitis posterior stärker.

Fig. e. Übergangsteil. Auch hier Degeneration der Py-S.Str. mit geringer schmaler Randdegeneration.

Fig. $f$ u. g. L.-W. 1 und Lendenanschwellung. Nur die PyS-Str. sind degeneriert.

Fall 2.

Fig. a bis g zeigen die Degeneration beschränkt auf die PyS-Str.; nur in der Cervikalanschwellung (a) greift die Degeneration etwas weiter nach vorn. Die Meningen sind nicht verdickt. 
Fig. 2 a.

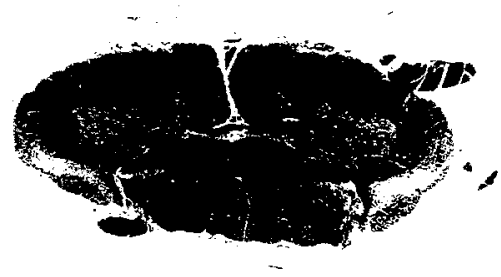

b.

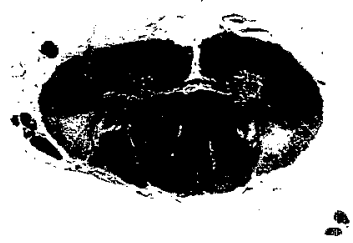

d.

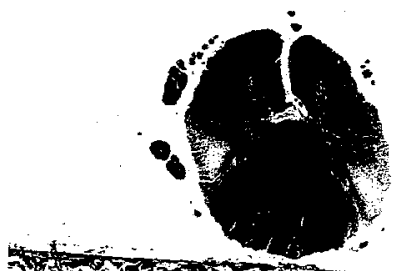

30 os

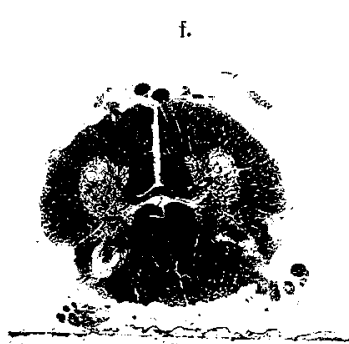

.

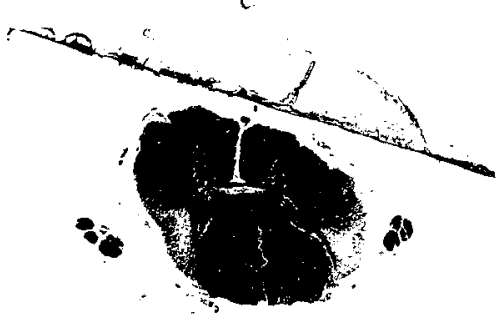

e.

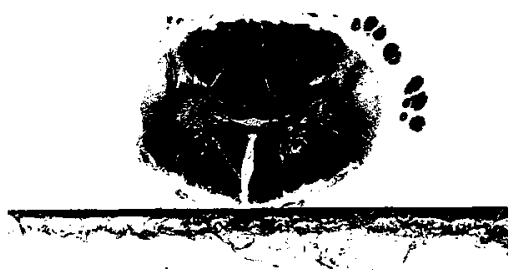

g

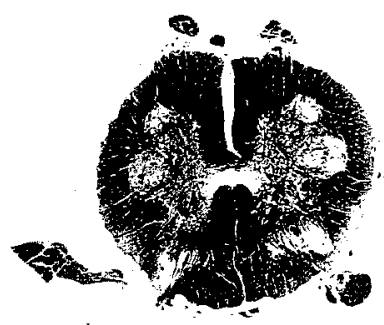

Fall 2. 